\title{
Radiosonde observations of vertical wave number spectra for gravity waves in the lower atmosphere over Central China
}

\author{
Shao Dong Zhang ${ }^{1,2}$, Chunming Huang ${ }^{1,2}$, and Fan Yi ${ }^{1,2}$ \\ ${ }^{1}$ School of Electronic Information, Wuhan University, Wuhan, People's Republic of China \\ ${ }^{2}$ Key Laboratory of Geospace Environment and Geodesy, Ministry of Education, Wuhan, People's Republic of China
}

Received: 18 August 2006 - Revised: 15 November 2006 - Accepted: 27 November 2006 - Published: 21 December 2006

\begin{abstract}
Vertical wave number spectra of inertial gravity waves in the troposphere and lower stratosphere over six stations at latitudes from $20^{\circ} \mathrm{N}$ to $40^{\circ} \mathrm{N}$ were statistically studied by using the data from Radiosonde observation on a twice daily basis at 08:00 and 20:00 LT. Statistically, the spectral characteristics seem to be independent of the local observation time, and show considerable conformity between the spectral of zonal and meridional kinetic energy densities. Compared with the spectra of the kinetic energy density, the spectra of the potential energy density are steeper. in addition the characteristic wave numbers of the spectra also show considerable consistency among the observations at different stations. As for the spectral slopes, they are systematically smaller (in magnitude) than the canon value of -3 , and exhibit slight height, seasonal and latitudinal variability. In addition to these universal characteristics, the spectral structures also exhibit departures and variations, and most of the departures and variations are related to the strong tropospheric jets. Generally, in the case of strong shear due to the tropospheric jet, there usually occur larger characteristic wave numbers and smaller spectral slopes. These departures seem to be persistent and climatological rather than transitory, indicating the significant impacts of the sheared background winds on the spectral structures of gravity waves.
\end{abstract}

Keywords. Meteorology and atmospheric dynamics (Middle atmosphere dynamics; Waves and tides; General or miscellaneous)

\section{Introduction}

Gravity waves (GWs) are ubiquitous and believed to be one of the most important waves in the stratosphere and mesosphere. They can impact significantly on local atmospheric

Correspondence to: Chunming Huang

(hcm720525@yahoo.com.cn) climatology (Alexander and Pfister, 1995; Alexander, 1998). Moreover, accompanying upward propagations, GWs transport energy and momentum from the lower atmosphere to the upper atmosphere, and deposit them in the mesosphere and lower thermosphere, resulting in a significant alteration of background atmospheric structure. In this way, GWs play a key role in determining global dynamics of the middle and upper atmosphere (Lindzen, 1981). Therefore, in order to comprehensively understand global mesospheric dynamics, it is of significant importance to study the character of atmospheric gravity wave activity.

Many characteristics of atmospheric GWs have been revealed by a great deal of observations, and a universal wave number spectrum is one of the most striking features of the atmospheric GWs, which means that for the vertical wave number (i.e. $m$ ) spectra of horizontal wind and temperature disturbances, the spectral slope and intensity remain nearly quasi-invariant. Since these spectral features appear to be independent of time and space, thus, the spectrum is said to be universal. The first observational evidence of the universal spectra of GWs was provided by VanZandt (1982). Subsequent observations (Nastrom et al., 1984; Nastrom and Gage, 1985) further confirmed that there were no systematic differences between the spectra over land and ocean, or during winter and summer. Since then, decades of nearly global observations by different instruments (e.g. radar, lidar, balloon, rocket and aircraft etc.) have revealed the characteristics of the atmospheric horizontal wind and temperature wave number spectra in more detail. Each spectrum has two different regions with distinct slopes. The spectral intensity at the small $m$ region seems to be proportional to $m$, while more important, its slope at the large $m$ region has a near-constant value of $q=-3$ (e.g. Dewan et al., 1984; Dewan and Good, 1986; Scheffler and Liu, 1986; Smith et al., 1987; Fritts et al., 1988; Tsuda et al., 1989; 1991; Wilson et al., 1990; Senft and Gardner, 1991; Fritts and VanZandt, 1993; Senft et al., 1993; Hostetler and Gardner, 1994; Eckermann, 1995b;

Published by Copernicus GmbH on behalf of the European Geosciences Union. 
1999; Bacmeister et al., 1996), and such a slope, though not always, tends to be roughly independent of time, location, and altitude. Between the low and high $m$ regions lies the knee of the spectrum, and the wave number corresponding to the knee is referred to as the characteristic vertical wave number $m_{*}$ (the vertical wave number of the most energetic gravity wave). Multiple data sets indicated $m_{*}$ was corresponding to a vertical wavelength of $\sim 2-5 \mathrm{~km}$ in the lower stratosphere and $\sim 10-30 \mathrm{~km}$ near the mesopause.

Many theories were proposed to account for the physical mechanism of the GW universal spectrum, e.g. 1) saturated spectrum theory (Dewan and Good, 1986; Smith et al., 1987); 2) Doppler-spread models (Hines, 1991, 1997a, b); and 3) diffusive models (e.g. Weinstock, 1976, 1990; Gardner, 1994; Zhu, 1994). In modeling studies of global scale atmospheric dynamics, the effects of medium and smallscale gravity wave drag (GWD) must be parameterized to obtain realistic mean atmospheric circulations and thermal structures. Based on the above cited theories, many spectrum models were developed to describe the GWD effects on the global atmospheric dynamics (e.g. Fritts and Lu, 1993; Hines, 1997a, b; Medvedev and Klaassen, 1995), and were extensively adopted in general circulation models (GCMs) (e.g. Hagan and Forbes, 2002, 2003; Medvedev et al., 1998; Manzini and McFarlane, 1998). Whereas, although these spectral models have been constrained to reproduce the "universal" $m^{-3}$ for the horizontal wind and temperature GW field energy spectra observed at high $m$, their corresponding GWD parameterizations can produce very different results (e.g. Dewan, 1994; Sato and Yamada, 1994). Furthermore, quantitative comparison between the GCMs and observations suggests that the GWD is a major source of uncertainties in model predictability and reliability (Fritts and Alexander, 2003). All these indicate the importance of more observations on wave number spectra of GWs.

Although there is surprising conformity of spectral amplitudes and slopes at the high $m$ region over a broad spatial coverage (Dewan and Good, 1986; Fritts and Chou, 1987; Smith et al., 1987; Fritts et al., 1988; Tsuda et al., 1989, 1990; Collins et al., 1994; Allen and Vincent, 1995), recent observations with higher-resolution at different locations and altitudes have on occasion revealed greater variations in the large $m$ portion of the spectra than models currently allow. The spectra at the large $m$ region in the stratosphere are often (although not always) less intense and shallower or steeper than expected (e.g. Shibata et al., 1988; Wilson et al., 1990; Tsuda et al., 1991, 1994; Senft et al., 1993; Hostetler and Gardner, 1994; Allen and Vincent; 1995). These features, when they arise, tend to be persistent and climatological rather than transitory (e.g. Tsuda et al., 1991). Most notably, these departures are usually observed in the stratosphere, and are accompanied with strong sheared wind (Eckermann, 1995a). Manuel and Caranti (2000) suggested that spectral slopes varied from -4 to -2 due to the effects of shearing background. All these lead to the natural conclusion that the wave number spectral features of GWs are far from fully understood, and more observations, especially in the troposphere and lower stratosphere (TLS), should be made.

The excellent height resolution (several tens to hundreds of meters) and relatively complete physical quantities of radiosonde observations permitted extensive studies of gravity waves in the TLS and their vertical wave number spectra (Tsuda et al., 1994; Shimizu and Tsuda, 1997; Vincent and Alexander, 2000; Yoshiki and Sato, 2000; Wang et al., 2005; Zhang and Yi, 2005, 2006). In each measurement by radiosonde, meteorological variables, such as pressure, temperature and relative humidity, are measured. The horizontal winds are attained by tracking the position of the balloon. The typical height coverage of the radiosonde observation is from the surface up to $25-30 \mathrm{~km}$, and the uncertainty of the upper height is due to the variable burst height of the balloon. The primary features of gravity waves in the TLS were revealed by many radiosonde observations (Vincent and Alexander, 2000; Wang et al., 2005; Zhang and Yi, 2005, 2006), which suggested that the dominant gravity wave disturbances in the TLS were inertial gravity waves.

The main purpose of this paper is to study the vertical wave number spectral character of inertial GWs in the TLS, including their universality and variability, especially the variability arising from the strong background wind shear. The data utilized in this paper are from radiosonde observations. These observations are based on routine measurements by meteorological agencies, which usually have a long-term (longer than 1 year) data set, and make it possible to study the seasonal variation of the vertical wave number spectra for inertial GWs. In order to investigate the geographical dependence of the spectral character, observational data from six stations at latitudes from $10^{\circ} \mathrm{N}$ to $40^{\circ} \mathrm{N}$ are included in our data set. A brief description of the data set and spectral calculation method are introduced in Sect. 2. In Sect. 3, we present the statistical results. Conclusions are given in the last section.

\section{Data description and methodology}

Radiosonde observations made by six meteorology stations on a twice daily basis at 08:00 and 20:00 LT were used in this study. These stations are Beijing $\left(116^{\circ} 28^{\prime} \mathrm{E} 39^{\circ} 4^{\prime} \mathrm{N}\right)$, Enshi $\left(109^{\circ} 28^{\prime} \mathrm{E}, 30^{\circ} 17^{\prime} \mathrm{N}\right)$, Yichang $\left(111^{\circ} 18^{\prime} \mathrm{E}, 30^{\circ} 42^{\prime} \mathrm{N}\right)$, Wuhan $\left(114^{\circ} 30^{\prime} \mathrm{E}, 30^{\circ} 40^{\prime} \mathrm{N}\right)$, Anqing $\left(117^{\circ} 03^{\prime} \mathrm{E}, 30^{\circ} 52^{\prime} \mathrm{N}\right)$ and Haikou $\left(110^{\circ} 12^{\prime} \mathrm{E}, 20^{\circ} 12^{\prime} \mathrm{N}\right)$. Their geographic locations are illustrated in Fig. 1. Available data sets used in this work from these stations with different observation durations are listed in Table 1. The raw data are sampled at an 8 -s interval, resulting in an uneven height resolution, which varies from tens to hundreds of meters. For convenience, the raw data were processed to have an even height resolution $(100 \mathrm{~m})$ by applying a linear interpolation. The maximum altitude of radiosonde observation is the burst height 
of the balloon. In our data set, about $58 \%$ of the measurements reached a height of $25 \mathrm{~km}$, but only about $35 \%$ reached $26 \mathrm{~km}$. Thus, we chose $25 \mathrm{~km}$ as the upper height limit of our analysis.

For extracting gravity wave disturbances, we should correctly remove the background winds and temperature from the raw data. In the presented work, the background fields $\left[u_{0}, v_{0}, T_{0}\right]$ are calculated by fitting a second-order polynomial to the vertical profiles of horizontal winds and temperature $[u, v, T]$, respectively. It should be emphasized here that, according to the theoretical analyses, in the saturation region $\left(m>m_{*}\right)$, the theoretical vertical wave number spectrum of gravity wave kinetic and potential energies, i.e. $E_{K}(m)$ and $E_{P}(m)$, can be respectively expressed as (Smith et al., 1987; Allen and Vincent, 1995; Fritts and Alexandre, 2003; and references herein):

$E_{K}(m) \approx \frac{p N^{2}}{10 \mathrm{~m}^{3}}$

and

$E_{P}(m) \approx \frac{N^{2}}{6 \mathrm{pm}^{3}}$,

where $N$ is the buoyancy frequency; $g$ is the gravitational acceleration $\left(g=9.8 \mathrm{~ms}^{-2}\right) ; p$ is the slope of the one-dimension frequency spectrum. The best estimate of $p$ from previous literature is $5 / 3$, and this value will be assumed hereinafter. Formulas (1) and (2) indicate that the buoyancy frequency $(N)$ controls the amplitudes of the power spectra. On the other hand, radiosonde observations over central China (Zhang and Yi, 2005, 2006) revealed that above the height of $18 \mathrm{~km}$, the buoyancy frequency $N$ is about 2 times that in the troposphere. Therefore, in our analyses, the secondorder polynomial fits are performed for two separate height ranges: a tropospheric segment from 2 to $10 \mathrm{~km}$, and a lower stratospheric segment from 18 to $25 \mathrm{~km}$.

Having removed the fitted background fields $\left[u_{0}, v_{0}, T_{0}\right]$ from the raw data $[u, v, T]$, the residuals are supposed to be GW perturbations $\left[u^{\prime}, v^{\prime}, T^{\prime}\right]$. Then, the zonal, meridional wave kinetic and potential energies per unit mass are computed from

$$
\begin{aligned}
& E_{K z}=\frac{1}{2} \overline{u^{\prime 2}}, \\
& E_{K m}=\frac{1}{2} \overline{v^{\prime 2}}
\end{aligned}
$$

and

$$
E_{P}=\frac{1}{2} \frac{\overline{g^{2} T^{\prime 2}}}{N^{2} T_{0}^{2}},
$$

respectively, where the over bar denotes an unweighted average over height. Subsequently, we perform a Lomb-Scargle (Scargle, 1982) spectral analysis on these wave energy densities (i.e. $E_{K z}, E_{K m}$ and $E_{P}$ ) to calculate the vertical wave

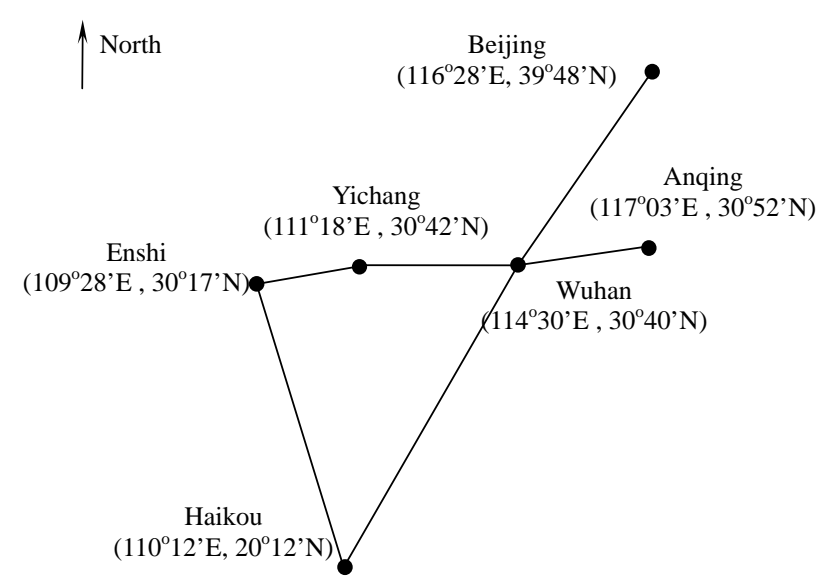

Fig. 1. Geographic locations of the radiosonde stations.

Table 1. Periods of available data set for each radiosonde station.

\begin{tabular}{ll}
\hline Stations & Periods of available data \\
\hline Beijing & $12 / 2001-6 / 2003$ \\
Enshi & $1 / 2001-12 / 2003$ \\
Yichang & $1 / 2001-12 / 2003$ \\
Wuhan & $1 / 2000-12 / 2004$ \\
Anqing & $1 / 2003-6 / 2004$ \\
Haikou & $1 / 2000-12 / 2004$ \\
\hline
\end{tabular}

number spectra for inertial GWs in the TLS. Finally, in the estimates of the spectral amplitudes, for the purpose of improving the confidence limits, the vertical wave number power spectra calculated from individual profiles are averaged, while in the calculation of the mean spectrum, in order to ensure that all spectra contribute equally to the shape of the mean spectrum, normalized individual power spectra are averaged as the mean spectrum (Allen and Vincent, 1995).

\section{Results}

\subsection{Averaged spectra}

Figure 2 illustrates the averaged vertical wave number spectra for the zonal wind kinetic energy (left panel), meridional kinetic energy (middle panel) and potential energy (right panel) in the troposphere (from 2 to $10 \mathrm{~km}$ ) over Wuhan station, and, as a comparison, the theoretical saturation spectra (Smith et al., 1987) are also plotted in Fig. 2. In the calculation of the theoretical spectrum (as expressed in formulas 1 and 2), the corresponding buoyancy frequencies $N$ are replaced by the values of $\bar{N}$, where $\bar{N}$ is the unweighted height and temporal average of the buoyancy frequency $N$. It is shown in Fig. 2 that each spectrum density shows two distinct regions, which are partitioned by spectral 
Table 2. Parameters of vertical wave number spectra for GWs in the troposphere and lower stratosphere over Wuhan station. $E_{K z}, E_{K m}$ and $E_{P}$ represent the zonal kinetic, meridional kinetic and potential energy densities, respectively. $q$ represents the slope of the vertical wavenumber spectrum in the large wave number regions. $m_{*}$ (in $($ Cycle $/ \mathrm{km})$ and $E_{*}\left(\mathrm{in}^{2} \mathrm{~s}^{2} /(\mathrm{Cycle} / \mathrm{km})\right)$ denote the characteristic vertical wave number and the corresponding spectral intensity.

\begin{tabular}{cccccccccc}
\hline \multirow{2}{*}{ Wuhan station } & \multicolumn{3}{c}{$E_{K z}$} & \multicolumn{4}{c}{$E_{K m}$} & \multicolumn{3}{c}{$E_{P}$} \\
\cline { 2 - 11 } & $q$ & $m_{*}$ & $E_{*}$ & $q$ & $m_{*}$ & $E_{*}$ & $q$ & $m_{*}$ & $E_{*}$ \\
\hline Troposphere & -2.32 & 0.47 & 3.55 & -2.31 & 0.47 & 3.22 & -2.74 & 0.50 & 4.69 \\
Lower stratosphere & -2.15 & 0.54 & 3.17 & -2.19 & 0.54 & 3.10 & -2.53 & 0.54 & 3.03 \\
\hline
\end{tabular}

Table 3. Parameters of vertical wave number spectra for GWs over Wuhan station at different local times.

\begin{tabular}{ccccccccccc}
\hline \multirow{2}{*}{ Wuhan station } & \multirow{2}{*}{ Local time } & \multicolumn{4}{c}{$E_{K z}$} & \multicolumn{4}{c}{$E_{K m}$} & \multicolumn{3}{c}{$E_{P}$} \\
\cline { 3 - 11 } & & $q$ & $m_{*}$ & $E_{*}$ & $q$ & $m_{*}$ & $E_{*}$ & $q$ & $m_{*}$ & $E_{*}$ \\
\hline \multirow{2}{*}{ Troposphere } & $08: 00$ & -2.34 & 0.47 & 3.59 & -2.32 & 0.47 & 3.34 & -2.70 & 0.47 & 4.83 \\
& $20: 00$ & -2.32 & 0.47 & 3.54 & -2.30 & 0.47 & 3.20 & -2.74 & 0.50 & 4.58 \\
\multirow{3}{*}{ Lower stratosphere } & $08: 00$ & -2.08 & 0.54 & 3.20 & -2.10 & 0.54 & 2.97 & -2.44 & 0.54 & 3.02 \\
& $20: 00$ & -2.24 & 0.54 & 3.16 & -2.30 & 0.54 & 3.27 & -2.63 & 0.54 & 3.08 \\
\hline
\end{tabular}
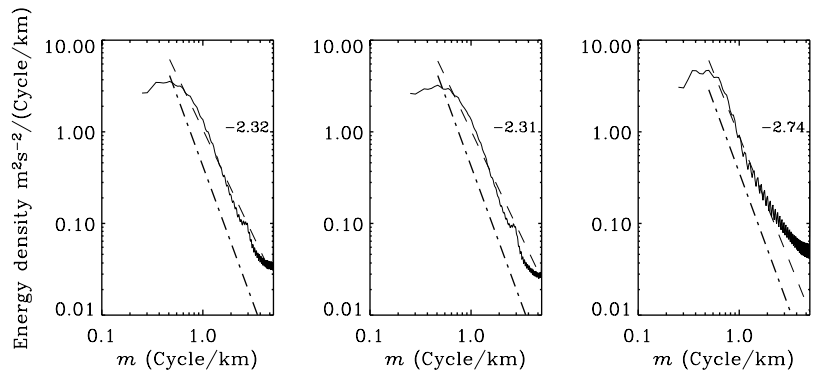

Fig. 2. Averaged vertical wave number spectra (solid curves) for the zonal kinetic energy density (left panel), meridional kinetic energy density (middle panel) and potential energy density (right panel) in the troposphere over Wuhan station, the dashed lines denote the fitted spectra. The theoretical saturation limits of Smith et al. (1987) are also plotted for comparison purposes (dash-dotted lines).

knees (characteristic wave number $m_{*}$ ). The characteristic wave numbers are estimated to be 0.47 and 0.50 Cycle $/ \mathrm{km}$ for the kinetic and potential energy densities, respectively, corresponding to the dominant vertical wavelengths of 4.3 and $4.0 \mathrm{~km}$, respectively, which are close to the dominant vertical wavelengths of gravity waves observed in the troposphere (Zhang and Yi, 2005, 2006), and the spectral amplitudes are generally larger than those of the theoretical saturation spectra. The maximum spectral intensities $E_{*}$ (the spectral intensity corresponding to the characteristic wave number) for the zonal and meridional kinetic energy densities are about 3.55 and $3.22 \mathrm{~m}^{2} \mathrm{~s}^{-2} /($ Cycle $/ \mathrm{km})$, respectively. At the
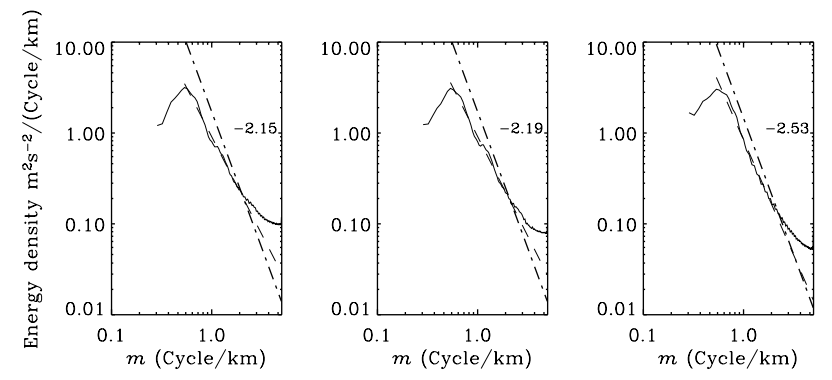

Fig. 3. Similar to Fig. 2, but for the averaged vertical wave number spectra in the lower stratosphere over Wuhan station.

small $m$ region $\left(m<m_{*}\right)$, spectral densities increase with the wave number. For the large $m$ region $\left(m>m_{*}\right)$, spectral densities decrease with the wave number, and the fitted slopes for the zonal and meridional kinetic energy densities are, respectively, -2.32 and -2.31 , which are smaller in magnitude (hereinafter, when we discuss the slope, we refer to its magnitude) than the canonical spectral slope of -3 . Compared with those of the kinetic energy, the spectrum of the potential energy density exhibits a larger $E_{*}\left(4.69 \mathrm{~m}^{2} \mathrm{~s}^{-2} /(\right.$ Cycle $\left./ \mathrm{km})\right)$ and steeper slope (-2.74).

Subsequently, we calculated the averaged vertical wave number spectra of GW energy density in the lower stratosphere (from 18 to $25 \mathrm{~km}$ ) over Wuhan station and plotted them in Fig. 3. Compared with those in the troposphere, the vertical wave number spectra in the lower stratosphere have larger characteristic wave numbers (smaller dominant 

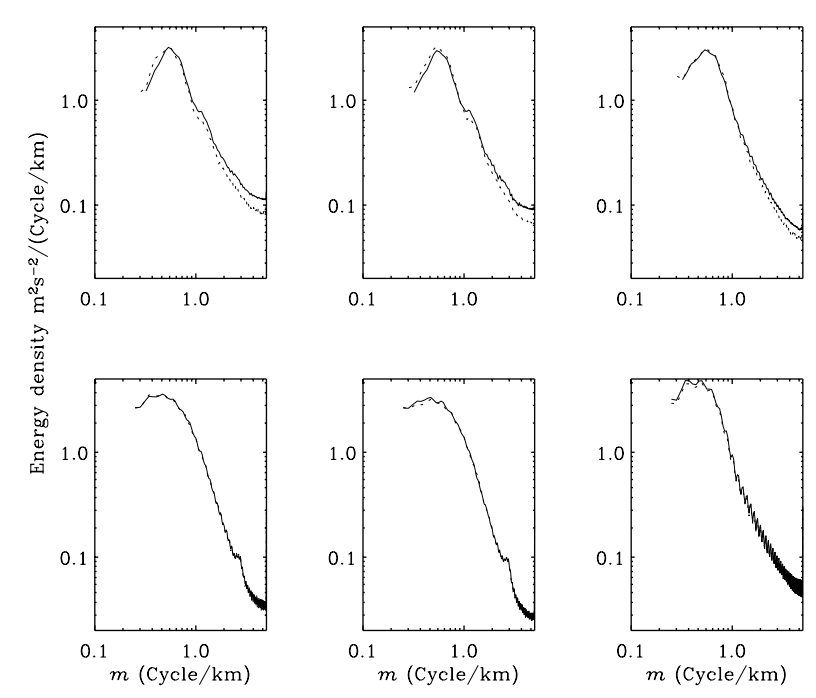

Fig. 4. Averaged vertical wave number spectra for the zonal kinetic energy density (left column), meridional kinetic energy density (middle column) and potential energy density (right column) in the troposphere (bottom row) and lower stratosphere (upper row) over Wuhan station. The solid and dotted curves denote the spectra at the local time of 08:00 and 20:00, respectively.

vertical wavelength), which is in good agreement with previous radiosonde observations (Zhang and Yi, 2005, 2006); and the spectral amplitudes in the lower stratosphere have smaller values and are generally smaller than those of the theoretical saturation spectra. As for the slopes, the fitted slopes for the zonal and meridional kinetic and potential energy densities are, respectively, -2.15 and -2.19 and -2.53 , which are smaller than the values in the troposphere. These differences reveal the significant impact of the strong wind shear induced by the tropospheric jet on GW spectral structures: the tropospheric jet induced Doppler shifting will lead to wave energy absorption by the background and an enhancement of GW activity with smaller scales.

The parameters of the vertical wave number spectra for GWs in the troposphere and lower stratosphere over Wuhan stations are listed in Table 2. Generally, the spectral features (i.e. the characteristic wave number $m_{*}$, maximum spectral intensity $E_{*}$, spectral slope $q$ ) of the vertical wave number spectra for zonal and meridional kinetic energy densities are in good agreement with each other, whereas compared with those of the kinetic energy, the spectrum of the potential energy density exhibits a steeper slope. Moreover, although the spectra amplitude for the potential energy density is larger than that for the kinetic energy density in the troposphere, the situation is reverse in the lower stratosphere. This result coincides with our previous observations (Zhang and $\mathrm{Yi}$, 2006).
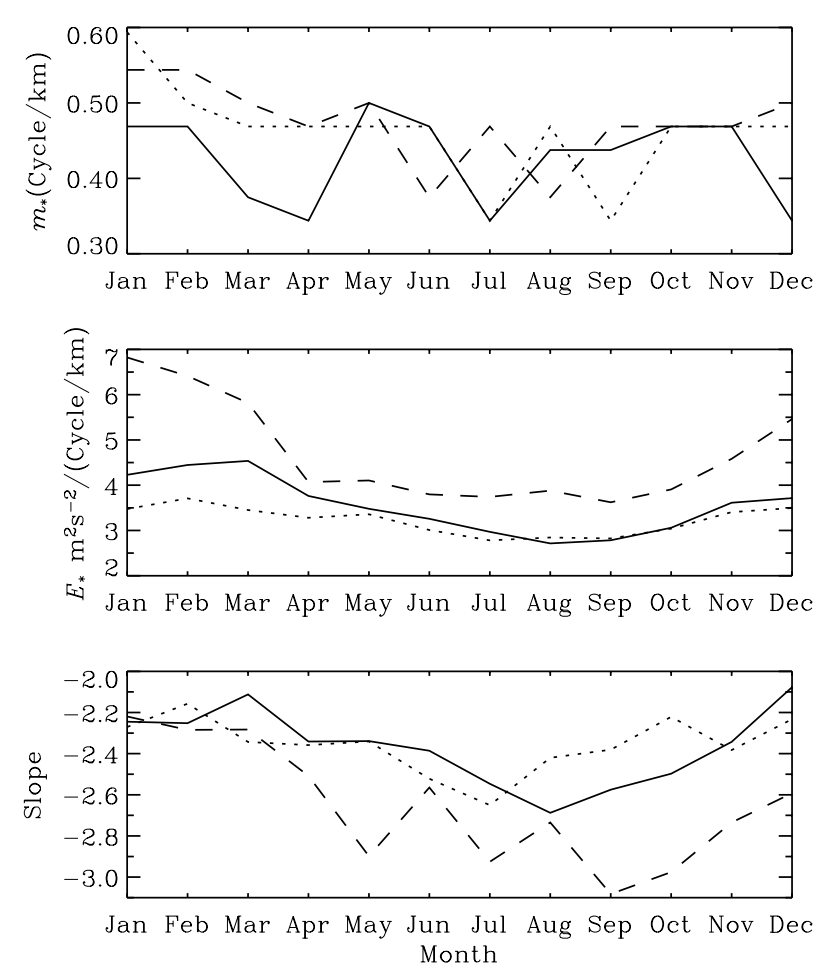

Fig. 5. Monthly variations of the characteristic wave numbers (upper panel), spectral amplitudes (middle panel) and slopes (bottom panel) of the mean vertical wave number spectra for GWs in the troposphere over Wuhan station. The solid, dotted and dashed curves denote the spectral parameters for the zonal kinetic energy, meridional kinetic energy and potential energy densities, respectively.

\subsection{Local time variability of wave number spectra}

Since our data were collected at two different local times (i.e. 08:00 LT and 20:00 LT), in this subsection, we want to investigate the differences in the wave number spectra acquired at these two local times. Figure 4 illustrates the averaged spectra at different local times at Wuhan station, and their primary spectral parameters are summarized in Table 3 . One can observe that, in both the troposphere and lower stratosphere, both the spectral shapes and spectral amplitudes at different local times are consistent with each other, indicating that the wave number spectra characteristics seem to be independent of the local time.

\subsection{Monthly variability of wave number spectra}

For the purpose of studying the monthly variability of the wave number spectra, we calculate individually the mean vertical wave number spectra for GWs in different months. Figures 5 and 6 demonstrate the monthly variations of the spectral parameters for the averaged vertical wave number spectra in the troposphere and stratosphere over Wuhan station, respectively. Several scenarios can be observed from 
Table 4. Parameters of vertical wave number spectrum for GWs over different stations.

\begin{tabular}{ccccccccccc}
\hline \multirow{2}{*}{ Stations } & \multicolumn{3}{c}{$E_{K z}$} & \multicolumn{3}{c}{$E_{K m}$} \\
\cline { 3 - 10 } & & $q$ & $m_{*}$ & $E_{*}$ & $q$ & $m_{*}$ & $E_{*}$ & $q$ & $m_{*}$ & $E_{*}$ \\
\hline \multirow{2}{*}{ Beijing } & Troposphere & -2.12 & 0.47 & 6.15 & -2.04 & 0.47 & 5.70 & -1.95 & 0.38 & 7.17 \\
& Lower stratosphere & -2.00 & 0.54 & 5.03 & -2.03 & 0.54 & 4.89 & -1.90 & 0.54 & 4.32 \\
& Troposphere & -2.29 & 0.47 & 3.76 & -2.29 & 0.47 & 3.32 & -2.35 & 0.34 & 5.70 \\
\multirow{4}{*}{ Yichang } & Lower stratosphere & -1.91 & 0.54 & 2.34 & -1.95 & 0.54 & 2.75 & -2.38 & 0.54 & 2.97 \\
\multirow{4}{*}{ Wuhan } & Troposphere & -2.18 & 0.47 & 3.60 & -2.17 & 0.47 & 3.25 & -2.32 & 0.34 & 5.29 \\
& Lower stratosphere & -2.11 & 0.54 & 2.97 & -2.12 & 0.54 & 3.02 & -2.42 & 0.54 & 3.07 \\
\multirow{2}{*}{ Anqing } & Troposphere & -2.32 & 0.47 & 3.55 & -2.31 & 0.47 & 3.22 & -2.74 & 0.50 & 4.69 \\
& Lower stratosphere & -2.15 & 0.54 & 3.17 & -2.19 & 0.54 & 3.10 & -2.53 & 0.54 & 3.03 \\
\multirow{2}{*}{ Haikou } & Troposphere & -2.25 & 0.47 & 3.43 & -2.17 & 0.47 & 3.25 & -2.40 & 0.34 & 4.30 \\
& Lower stratosphere & -2.11 & 0.54 & 3.47 & -2.10 & 0.50 & 3.31 & -2.27 & 0.39 & 3.31 \\
& Troposphere & -2.47 & 0.47 & 2.68 & -2.43 & 0.47 & 2.96 & -3.02 & 0.47 & 4.00 \\
& Lower stratosphere & -2.23 & 0.54 & 2.84 & -2.29 & 0.57 & $2-.65$ & -2.37 & 0.54 & 3.19 \\
\hline
\end{tabular}

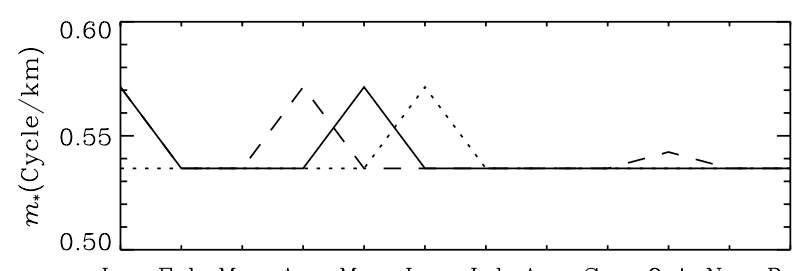

Jan Feb Mar Apr May Jun Jul Aug Sep Oct Nov Dec
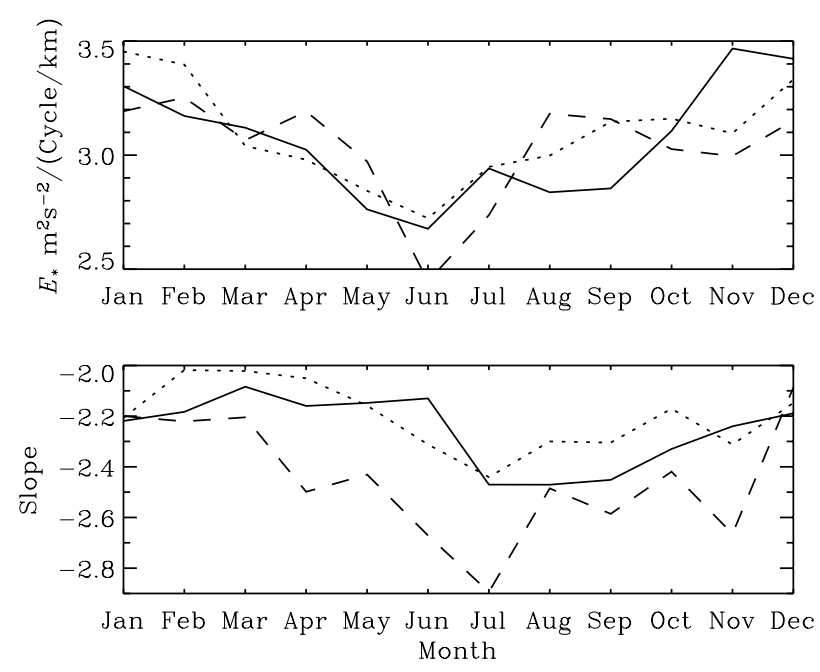

Fig. 6. Similar to Fig. 5, but for the monthly variations of the spectral parameters of the mean vertical wave number spectra for GWs in the lower stratosphere.

these figures: 1) there are no obvious monthly variations of the characteristic wave numbers, and the characteristic wave numbers in the troposphere are smaller than those in the lower stratosphere, which is due to the tropospheric jet induced Doppler shifting. 2) the spectra amplitudes in the troposphere are larger than those in the lower stratosphere.
Moreover, the spectral magnitudes exhibit evident seasonal cycle: larger and smaller spectral amplitudes usually occur in winter and summer, respectively. These seasonal cycles are consistent with that of the tropospheric jet, indicating the important role of the tropospheric jet in determining the wave number spectra of GWs in the lower atmosphere. 3) as for the spectral slopes, their magnitudes in winter are usually smaller than those in summer. Considering that the strong tropospheric jet usually occurs in winter, this result essentially reflects the significant effects of the wind shear on the wave number spectra of GWs, and the spectra in the lower stratosphere are generally shallower than those in the troposphere. This difference can also be accounted from that the tropospheric jet may lead to the enhancement of GWs with smaller vertical scales by the Doppler shifting effects. Finally, it seems that the slopes of the vertical wave number spectra for the potential energy density are generally larger than those for the kinetic energy density.

\subsection{Geographical variability of wave number spectra}

In order to study the geophysical variability of the vertical wave number spectra, we calculated the averaged wave number spectra and summarized their primary spectra parameters in Table 4. The characteristic wavenumbers are almost independent of the locations, except that larger values for the potential energy densities in the troposphere over Yichang and Haikou stations and a smaller value for the potential energy density in the lower stratosphere over Anqing were observed. The latitudinal variations of the maximum spectral intensities seem generally consistent with those of the magnitudes of the tropospheric jets, which has been reported by Zhang and Yi (2006). An exception is over Beijing station, where much larger $E_{*}$ can be observed, which may be due to the fact that gravity wave activity in the lower atmosphere is more intensive in winter than in other seasons because of 
the larger tropospheric jet (Zhang and Yi, 2005, 2006), and in our data set from Beijing station, more observational data in winter is included (as shown in Table 1). Therefore, the latitudinal distribution of $E_{*}$ revealed the impact of the tropospherc jet on GW activity. As for the slopes of the spectra over different stations, they are generally smaller than -3 (an exception is over Haikou sation) and show slight latitudinal variations. The largest value of the slopes occurs at Haikou, which has the weakest tropospheric jet (Zhang and Yi, 2006), and an extremely small value of -1.90 was observed for the potential energy density over Beijing.

Except the geophysical variability, Table 4 demonstrates some consistent spectral characteristics over different stations, which are summarized as follows: 1) the characteristic wave numbers show considerable geophysical consistency, which has been reported by Zhang and Yi (2006), and due to the effect of the tropospheric jet induced strong wind shear, the dominant vertical scale for GWs in the lower stratosphere are smaller than that in the troposphere. 2) The spectral characteristics (including the characteristic wave number, spectral amplitude and spectral slope) of the zonal and meridional kinetic energy densities are in good agreement with each other, while the spectra of the potential energy density are usually steeper than those of the kinetic energy density. 3) The spectral amplitudes in the troposphere and lower stratosphere are larger and smaller than the theoretical saturation spectral amplitudes (Smith et al., 1987), respectively (not presented here). 4) The spectral slopes are systematically smaller than the canon value of -3 , and show slight height, seasonal and latitudinal variability, and the spectra in the stratosphere are statistically shallower than those in the troposphere.

\section{Conclusions}

Vertical wave number spectra of GWs in the TLS are statistically studied by using the data from Radiosonde observation made by the Beijing, Enshi, Yichang, Wuhan, Anqing and Haikou Meteorological agencies on a twice daily basis at 08:00 and 20:00 LT. The primary results are summarized as follows.

The characteristic wave numbers show considerable geophysical consistency, and considerable conformity between the spectral character of the zonal and meridional kinetic energy densities is also seen. As for the spectral slopes, they are systematically smaller than the canon value of -3 , and exhibit slight height, seasonal and latitudinal variability. Additionally, the slopes for the potential energy density are statistically larger than those for the kinetic energy densities. Moreover, generally, the spectra characteristics seem to be independent of the local observation time.

Except the conformity, the vertical wave number spectra also exhibit variations and departures, and these departures are climatological and persistent rather than transitory. The characteristic wave numbers in the troposphere are obviously smaller than those in the lower stratosphere. For the spectral amplitudes, a quantitative comparison indicates that the spectra amplitudes in the troposphere are statistically larger than the theoretical saturation spectral amplitudes (Smith et al., 1987) and those in the lower stratosphere, while the spectral amplitudes in the lower stratosphere are generally smaller than the theoretical saturation spectral amplitudes (Smith et al., 1987). More interesting, the mean spectral slopes in the lower stratosphere, in winter and in the middle latitudinal region, usually have smaller values. Most of the abovementioned variations can be explained by the strong tropospheric jet induced Doppler shifting, which indicates the important role of wind shear in determining the vertical wave number spectral character of GWs. Generally, the strong shear due to the tropospheric jet tends to decrease the dominant wavelength, spectra amplitude and slope.

Acknowledgements. We wish to thank the anonymous reviewers for their valuable suggestions on this paper. This work was jointly supported by the National Natural Science Foundation of China through grant 40336054 and 40575020, the Program for New Century Excellent Talents in University of China and the Cultivation Fund of the Key Scientific and Technical Innovation Project, Ministry of Education of China (No: 2004-295).

Topical Editor U.-P. Hoppe thanks G. G. Shepherd and another referee for their help in evaluating this paper.

\section{References}

Alexander, M. J.: Interpretations of observed climatological patterns stratospheric gravity wave variance, J. Geophys. Res., 103, 8627-8640, 1998.

Alexander, M. J. and Pfister, L.: Gravity wave momentum flux in the lower stratosphere over convection, Geophys. Res. Lett., 22, 2029-2032, 1995.

Allen, S. J. and Vincent, R. A.: Gravity-wave activity in the lower atmosphere: Seasonal and latitudinal variations, J. Geophys. Res., 100, 327-1350, 1995.

Bacmeister, J. T., Eckermann, S. D., Newman, P. A., Lait, L., Chan, K. R., Loewenstein, M., Proggitt, M. H., and Gary, B. L.: Stratospheric horizontal wavenumber spectra of winds, potential temperature and atmospheric tracers observed by high-altitude aircraft, J. Geophys. Res., 101, 9411-9470, 1996.

Collins, R. L., Nomura, A., and Gardner, C. S.: Gravity waves in the upper mesosphere over Antactica: Lidar observations at the South pole and Syowa, J. Geophys. Res., 99, 5475-5485, 1994.

Dewan, E. M.: The saturated-cascade model for atmospheric gravity wave spectra, and the wavelength-period (W-P) relations, similitude theory of gravity wave spectra, Geophys. Res. Lett., 21, 817-820, 1994.

Dewan, E. M. and Good, R. E.: Saturation and the "universal" spectrum for vertical profiles of horizontal scalar winds in the atmosphere, J. Geophys. Res., 91, 2742-2748, 1986.

Dewan, E. M., Grossbard, N., Quesada, A. F., and Good, R. E.: Spectral analysis of $10 \mathrm{~m}$ resolution scalar velocity profiles in the stratosphere, Geophys. Res. Lett., 11, 80-83, 1984. 
Eckermann, S. D.: Effects of background winds on vertical wavenumber spectra of atmospheric gravity waves, J. Geophys. Res., 100, 14 097-14 112, 1995a.

Eckermann, S. D.: On the observed morphology of gravity-wave and equatorial-wave variance in the stratosphere, J. Atmos. Terr. Phys., 57, 105-134, 1995b.

Eckermann, S. D.: Isentropic advection by gravity waves: quasiuniversal $M^{-3}$ vertical wavenumber spectra near the onset of instability, Geophys. Res. Lett., 26, 201-204, 1999.

Fritts, D. C. and Alexander, M. J.: Gravity wave dynamics and effects in the middle atmosphere, Rev. Geophys., 41(1), 1003, doi:10.1029/2001RG000106, 2003.

Fritts, D. C. and Chou, H. G.: An investigation of the vertical wavenumber and frequency spectra of gravity waves in the lower stratosphere, J. Atmos. Sci., 44, 3610-3624, 1987.

Fritts, D. C. and Lu, W.: Spectral estimate of gravity wave energy and momentum flux, Part II: Parameterization of wave forcing and variability. J. Atmos. Sci., 50, 3695-3713, 1993.

Fritts, D. C, Tsuda, T., Sato, T., Fukao, S., and Kato, S.: Observational evidence of a saturated gravity wave spectrum in the troposphere and lower stratosphere, J. Atmos. Sci., 45, 1741-1759, 1988.

Fritts, D. C. and VanZandt, T. E.: Spectral estimate of gravity wave energy and momentum flux, Part I: Energy dissipation, acceleration and constrains, J. Atmos. Sci., 50, 3685-3694, 1993.

Gardner, C. S.: Diffusive filtering of gravity wave spectra in the atmosphere, J. Geophys. Res., 99, 20 601-20 622, 1994.

Hagan, M. E. and Forbes, J. M.: Migrating and nonmigrating diurnal tides in the middle and upper atmosphere excited by tropospheric latent heat release, J. Geophys. Res., 107(D24), 4754, doi:10.1029/2001JD001236, 2002.

Hagan, M. E. and Forbes, J. M.: Migrating and nonmigrating semidiurnal tides in the upper atmosphere excited by tropospheric latent heat release, J. Geophys. Res., 108(A2), 1062, doi:10.1029/2002JA009466, 2003.

Hines, C. O.: The saturation of gravity waves in the middle atmosphere, Part II: Development of Doppler-spread theory, J. Atmos. Sci., 48, 1360-1379, 1991.

Hines, C. O.: Doppler-spread parameterization of gravity-wave momentum deposition in the middle and upper atmosphere, 1, Basic formulation, J. Atmos. Sol. Terr. Phys., 59, 371-386, 1997a.

Hines, C. O.: Doppler-spread parameterization of gravity-wave momentum deposition in the middle and upper atmosphere, 2, Broad and quasi monochromatic spectra, and implementation, J. Atmos. Sol. Terr. Phys., 59, 387-400, 1997 b.

Hostetler, C. A. and Gardner, C. S.: Observations of horizontal and vertical wave number spectra of gravity wave motions in the stratosphere and mesosphere over the mid-Pacific, J. Geophys. Res., 99, 1283-1302, 1994.

Lindzen, R. S.: Turbulence and stress owing to gravity wave and tidal breakdown, J. Geophys. Res., 86, 9707-9714, 1981.

Manuel, P. and Caranti, G.: Power spectrum of a gravity wave propagating in a shearing background wind, Geophys. Res. Lett., 27, 101-104, 2000.

Manzini, E. and McFarlane, N.: The effect of varying the source spectrum of a gravity wave parameterization in a middle atmosphere general circulation model, J. Geophys. Res., 103, 31 523$31539,1998$.

Medvedev, A. and Klaassen, G.: Vertical evolution of gravity wave spectra and the parameterization of associated wave drag, J. Geophys. Res., 100, 25 841-25 853, 1995.

Medvedev, A., Klaassen, G., and Beagley, S.: On the role of an anisotropic gravity wave spectrum in maintaining the circulation of the middle atmosphere, Geophys. Res. Lett., 25, 509-512, 1998.

Nastrom, G. D. and Gage, K. S.: A climatology of atmospheric wavenumber spectra of wind and temperature observed by commercial aircraft, J. Atmos. Sci., 42, 950-960, 1985.

Nastrom, G. D., Gage, K. S., and Jasperson, W. H.:, The atmospheric kinetic energy spectrum, $10^{0}-10^{4} \mathrm{~km}$, Nature, 310, 3638, 1984.

Sato, K. and Yamada, M.: Vertical structure of atmospheric gravity waves revealed by the wavelet analysis, J. Geophys. Res., 99, 20 623-20 631, 1994.

Scargle, J. D.: Studies in astronomical time series analysis II, statistical aspects of spectral analysis of unevenly spaced data, Astrophys J., 263, 835-853, 1982.

Scheffler, A. O. and Liu, C. H.: The effects of Doppler shift on the gravity waves spectra observed by MST radar, J. Atmos. Terr. Phys., 48, 1225-1231, 1986.

Senft, D. C. and Gardner, C. S.: Seasonal variability of gravity wave activity and spectra in the mesopause region at Urbana, J. Geophys. Res., 96, 17 229-17 264, 1991.

Senft, D. C., Hostetler, C. A., and Gardner, C. S.: Characteristics of gravity wave activity and spectra in the upper stratosphere and upper mesosphere at Arecibo during early April 1989, J. Atmos. Terr. Phys., 55, 425-439, 1993.

Shibata, T., Ichimori, S., Narikiyo, T., and Maeda, M.: Spectral analysis of vertical temperature profiles observed by a lidar in the upper stratosphere and the lower mesosphere, J. Meteorol, Soc., Jpn., 66, 1001-1005, 1988.

Shimizu, A. and Tsuda, T.: Characteristics of Kelvin waves and gravity waves observed with radiosondes over Indonesia, J. Geophys. Res., 102, 26 159-26 171, 1997.

Smith, S. A., Fritts, D. C., and VanZandt, T. E.: Evidence for a saturated spectrum of atmospheric gravity waves, J. Atmos. Sci., 44, 1404-1410, 1987.

Tsuda, T., Inoue, T. , Fritts, D. C., VanZandt, T. E., Kato, S., and Fukao, S.: MST radar observations of a saturated gravity wave spectrum, J. Atmos. Sci., 46, 2440-2447, 1989.

Tsuda, T., Kato, S., Yokoi, T., Inoue, T., and Yamamoto, M.: Gravity waves in the mesosphere observed with the middle and upper atmosphere radar, Radio Sci., 25, 1005-1018, 1990.

Tsuda, T., Murayama, Y., Wiryosumarto, H., Harijono, S. W. B., and Sato, S.: Radiosonde observations of equatorial atmosphere dynamics over Indonesia, 2, Characteristics of gravity waves, J. Geophys. Res., 99, 10 491-10 506, 1994.

Tsuda, T., VanZandt, T. E., Mizumoto, M., Kato, S., and Fukao, S.: Spectral analysis of temperature and Brunt-Väisälä freuquency frequency fluctuations observed by radiosondes, J. Geophys. Res., 96, 17 265-17 278, 1991.

VanZandt, T. E.: A universal spectrum of buoyancy waves in the atmosphere, Geophys. Res. Lett., 9, 575-578, 1982.

Vincent, R. A. and Alexander, M. J.: Gravity waves in the tropical lower stratosphere: An observational study of seasonal and interannual variability, J. Geophys. Res., 105, 17 971-17 982, 2000.

Vincent, R. A., S. J. Allen, and S. D. Eckermann: Gravity wave parameters in the lower stratosphere, in: Gravity Wave Pro- 
cesses: Their Parameterization in Global Climate models, edited by: Hamilton, K.,NATO ASI Ser. I, 50, 7-25, 1997.

Wang L., Geller, M. A., and Alexander, M. J.: Spatial and temporal variations of gravity wave parameters. Part I: Intrinsic frequency, wavelength, and vertical propagation direction, J. Atmos. Sci., 62, 125-142, 2005.

Weinstock, J.: Nonlinear theory of acoustic-gravity waves Part I: Saturation and enhanced diffusion, J. Geophys. Res., 81, 633652, 1976.

Weinstock, J.: Saturate and unsaturated spectra of gravity waves and scale-dependent diffusion, J. Atmos. Sci., 47, 2211-2225, 1990.

Wilson, R., Hauchecorne, A., and Chanin, M. L.: Gravity wave spectra in the middle atmosphere as observed by Rayleigh lidar, Geophys. Res. Lett., 17, 1585-1588, 1990.
Yoshiki, M. and Sato, K.: A statistical study of gravity waves in the polar regions based on operational radiosonde data, J. Geophys. Res., 105, 17 995-18 011, 2000.

Zhang, S. D. and Yi, F.: A statistical study of gravity waves from radiosonde observations at Wuhan $\left(30^{\circ} \mathrm{N}, 114^{\circ} \mathrm{E}\right)$, China, Ann. Geophys., 23, 665-673, 2005, http://www.ann-geophys.net/23/665/2005/.

Zhang, S. D. and F. Yi, F.: Latitudinal and seasonal variations of inertial gravity wave activity in the lower atmosphere over central China, J. Geophys. Res., in press, 2006.

Zhu, X.: A new theory of saturated gravity wave spectrum in the middle atmosphere, J. Atmos. Sci., 51, 3615-3626, 1994. 